\title{
INTERDISCIPLINARIEDAD, CIENCIAS SOCIALES Y ODONTOLOGÍA SOCIAL, PREVENTIVA Y SANITARIA
}

\section{María Susana AZCONA ${ }^{1}$}

\section{Resumen}

La autora retoma algunos conceptos claves de la epistemología de Pierre Bourdieu y de la perspectiva crítica de Héctor Vázquez para construir el espacio teórico de la Odontología Social, Preventiva y Sanitaria.

Palabras claves: Epistemología de las ciencias sociales - InterdisciplinariedadOdontología Social, Sanitaria y Preventiva

\begin{abstract}
The author takes up some key concepts from the epistemology of Pierre Bourdieu and from the critical perspective of Hector Vazquez to build up the theoretical space of the Preventive and Sanitary Social Odontology.
\end{abstract}

Key words: Epistemology of social sciences -Interdisciplinary - Preventive and Sanitary Social Odontology.

\section{Resumé}

L'auteur reprend quelques concepts clés de l'épistémologie de Pierre Bourdieu et du point de vue critique de Héctor Vázquez pour construire l'espace théorique de 1 'Odontologie Préventive, Sociale et Sanitaire.

Mots clé: Épistémologie des sciences sociales-Interdisciplinarité-Odontologie Préventive, Sociale et Sanitaire.

\footnotetext{
${ }^{1}$ Investigadora Principal CIUNR y ex Profesora titular de la catadora Odontología Social I de la Facultad de Odontología de la UNR. Argentina

Fecha de recepción del artículo: Mayo 2016

Fecha de evaluación: Agosto 2016
} 
A diferencia de la categoría de multidisciplinariedad, la que supone el abordaje colindante de distintas disciplinas sobre una o más cuestiones a investigar, el concepto de Interdisciplina o de Interdisciplinariedad, refiere a la concurrencia de distintas disciplinas en varios núcleos de convergencia teórico-metodológicas, construyendo de tal manera, un denominador teórico común para la delimitación de una problemática de investigación. (Leo Apostel y Guy Michaud 1975). Por lo tanto, dichos conceptos tienen exclusivamente sentido durante el proceso de investigación.

Tal base epistemológica puede postularse para todas las ciencias. Es decir, tanto para las ciencias físico-naturales como para las ciencias sociales. Aunque, claro está, existan especifidades epistemológicas inherentes a cada una de ellas.

Conviene destacar, que son los investigadores quienes durante el proceso de investigación construyen las modalidades específicas de convergencia interdisciplinar de acuerdo a las características de la problemática concreta de investigación. Este postulado lo retomaremos más adelante cuando tratemos el tema de la Odontología Social, Preventiva y Sanitaria como construcción interdisciplinar.

La postura epistemológica de P. Bourdieu es muy conocida, su relevancia en el dominio de las ciencias sociales nos obliga a presentar, aunque sucintamente, sus aspectos centrales. Su perspectiva se articula alrededor de los siguientes conceptos clave: campo, habitus, capital científico, autonomía y reflexividad.

En efecto, colocando en tela de juicio el concepto de comunidad científica, Bourdieu lo suplanta por el de campo. Un concepto multidimensional puesto que se aplica a varios dominios: religioso, filosófico, estético, etc. y, por supuesto, también a la ciencia. El concepto de campo científico posee características compartidas en los dominios mencionados. Es presentado como un ámbito de posiciones encontradas que se enfrentan entre sí, defendiendo intereses que no tienen nada que ver con la producción científica estrictamente considerada. El científico procura posicionarse en el campo específico de su especialidad buscando prestigio y autoridad que se transforman en capital científico. (Bourdieu 1999. 84, 85 y 90, 91). 
Por lo tanto, tales intereses vehiculizan valoraciones ideológicas y prejuicios. Esto hace que dicho campo sea arbitrario. No hay neutralidad valorativa ni ciencia neutra. Lo que sí existe, a partir del concepto de reflexividad, son consideraciones metodológicas fundamentadas. La reflexividad, que opera entonces a modo de fundamentación crítica, adquiere un sentido de conciencia y autoconciencia epistemológica (Francisco Vázquez García 2006).

"La reflexividad es un imperativo que no concierne al investigador individual, sino al conjunto del campo sociológico. El proyecto de Bourdieu y de su equipo pretende hacer que el control reflexivo ejercido mutuamente y a escala, a ser posible, internacional, desempeñe en las ciencias sociales el mismo papel que las ciencias naturales asignan a técnicas como la reduplicación experimental o la formalización. Estos son instrumentos que hacen operativo todo el capital de conceptos, artefactos y métodos acumulados históricamente en el campo, con objeto de despersonalizar, desparticularizar los resultados obtenidos, logrando así su validación colectiva”. (Francisco Vázquez García 2006).

La arbitrariedad se sobredimensiona en el campo científico de las ciencias sociales, en el que los grados de autonomía se reducen en relación a la ambigüedad que lo define, se construye un arco de posibilidades que van de lo "científico" a lo político y viceversa.

El campo científico de las ciencias sociales se encuentra poblado de obstáculos sociales que de diversos modos restringen la autonomía del quehacer científico, y, por lo tanto, de la producción de conocimientos científicos. Esto es así en las instituciones en las que se hace ciencia social, en las modalidades de obtener recursos para el financiamiento de proyectos y equipos de investigación, y para la publicación de los resultados obtenidos en revistas especializadas de jerarquía.Por otro lado, los científicos se agrupan con el propósito de luchar por el monopolio del conocimiento científico. Los científicos que tienen posiciones de poder al interior de un campo científico, tienden a reproducirse a través del lobby y el reclutamiento. Quienes pertenecen al establisment imponen sus modalidades de hacer ciencia, y la defienden contra los grupos de científicos que disputan su poder. 
"En efecto, el working cosensus de una ortodoxia fundada en la complicidad social de los doctores tiende a ejercer una censura social (disfrazada de control científico), ya sea de manera totalmente directa a través de las prohibiciones, a veces explícitas, en materias de publicaciones y de citación, ya sea más secretamente a través de procedimientos de reclutamiento que, al privilegiar- por el funcionamiento en red y el lobbying- los criterios sociales más o menos maquillados como criterios científicos o académicos, tienden a reservar el nombramiento en posiciones favorables para la producción, y por ello, para la competencia científica, a ciertas categorías de agentes definidos en términos puramente sociales, titulares de ciertos diplomas prestigiosos ocupantes de ciertas posiciones sociales en la enseñanza o la investigación, o, a la inversa, a excluir a priori ciertas categorías, mujeres, jóvenes, o extranjeros, por ejemplo" (1999:118).

Esto acontece en todo campo científico y a nivel nacional e internacional. Aunque la internalización del conocimiento permite, en alguna medida, ampliar el espacio de control crítico del conocimiento.

Es el momento de aclarar, que el concepto de habitus refiere a los modos de hacer y comportase dentro de un campo científico, a costumbres y modalidades adquiridas en los procesos de producción de conocimiento de cada campo disciplinar que los científicos que los integran deben respetar.

¿Cómo entiende P. Burdieu el concepto de interdisicplinariedad?

En la entrevista que con J. Heilbron y B. Maso Bourdieu responda a la siguiente pregunta: "En la sociología, hay una tendencia muy grande a la especialización, a veces excesiva. ¿Es también un aspecto del efecto Gerrschenkron del que acaba de hablar?”

Respuesta: "Absolutamente. Se quiere imitar a las ciencias avanzadas ${ }^{2}$ donde las personas tienen objeto de investigación muy preciso y muy pequeño. Esta

\footnotetext{
${ }^{2}$ ¿Por qué dice Bourdieu avanzadas? ¿Será porque mantiene la concepción positivista de Comte que se expresa también en Durkheim? ¿Un despliegue unilineal del pensamiento que va de lo inferior a lo superior y de lo simple a lo complejo? En La Reglas del método sociológico Durkheim sostiene que las ciencias sociales son inmaduras en relación a las ciencias físiconaturales. En realidad se trata de desarrollos diferenciados a partir de contextos metodológicos muy diferentes.
} 
especialización excesiva que exalta el modelo positivista, por una especie de sospecha con respecto a toda ambición general, percibida como un vestigio de ambición globalizante de la filosofía. En realidad, estamos todavía en una fase en la cual es absurdo separar, por ejemplo, la sociología de la educación y la sociología de la cultura. ¿Cómo puede hacerse sociología de la literatura o sociología de la ciencia sin referencia a la sociología del sistema escolar? Por ejemplo, cuando se hace una historia social de los intelectuales, se olvida casi siempre tomar en cuenta la evolución estructural del sistema escolar, que puede conducir a efectos de "superproducción" de diplomados, inmediatamente traducidos al campo intelectual, tanto al nivel de la producción- con la aparición, por ejemplo, de una bohemia social e intelectualmente subversiva-como al nivel del consumo- con la transformación cualitativa y también cuantitativa del publico de lectores-. Evidentemente esta especialización responde también a intereses". P. Bourdieu (1993:48).

Parece muy claro que la separación disciplinar en especializaciones y superespecializaciones supone, en la visión de P. Bourdieu, una barrera que necesita superarse. Él lo consigue mediante la articulación de sus conceptos de habitus y de reflexibilidad. Ellos le permiten trascender la actividad práctica de lo teórico, lo subjetivo de lo objetivo, lo monológico de lo dialógico y también, sin duda, los limites disciplinares.

Entre quienes plantean la problemática que nos ocupa desde una perspectiva epistemológica situada en tiempo, lugar e ideología (en el sentido más amplio del término), Héctor Vázquez persiste en su intento de construir una línea de pensamiento histórica y crítica. Insiste, entre otros (1986), en afirmar que las ciencias sociales son poliparadigmáticas y, no como lo sostiene Khun (1980) preparadigmáticas. Esto es así puesto que la diversidad de concepciones teóricas no introduce una nueva barrera epistemológica, en tanto se considere la existencia de una inconmensurabilidad débil Vázquez (1986 y 1994).

Entroncándonos en la línea epistemológica propuesta por este autor (1984, 1986, 1988, 1994, 2000, 2005, 2014), asumimos las ciencias sociales como una red multidimensional conectada entre sí por núcleos de convergencia interdisciplinar. Los que, a su vez, conforman cada especificidad disciplinar. A modo de ejemplo: la 
antropología sociocultural se constituye mediante la convergencia de la etnología, de la sociología, de la historia, y de la etnolinguiística. Mientras que en la constitución de la etnolingüística o lingüística antropológica, en la medida que estudia las relaciones entre la lengua y la etnia (Fernández Guizzetti 1981), y por lo tanto, las interacciones entre lengua sociedad, pensamiento, cultura y percepción, emana de la convergencia de la sociolinguiística, la antropología sociocultural, la etnología y obviamente la semiología (Vázquez- Bigot (2006 y 2013).

Apoyándose críticamente en las posturas epistemológicas de L. Goldman, G. Balandier, L. V. Thomas, P. Anderson, P. Bourdieu y Hempel se propone aportar elementos con el propósito de contribuir a la construcción de una antropología socio cultural crítica y emancipadora Vázquez (1984, 1992, 1994,2000 y 2005).

"El propósito fundamental de la Antropología Emancipadora es el de poner en evidencia, primero, e investigar, después, las situaciones histórico - culturales y socio -económicas de opresión realmente existentes en las distintas sociedades. Por supuesto que el desmontaje teórico de tales circunstancias tiene una finalidad deliberada: el interés y la intención de diseñar políticas socio - culturales específicas racionalmente fundamentadas que permitan trascender dichas situaciones objetivas de opresión.

El principal componente de la Antropología Emancipadora es su carácter crítico en un doble sentido:

a) la evaluación de las diferentes posturas teóricas planteadas dentro de un contexto problemático mediante un razonamiento capaz de discriminar matices argumentativos y de colocar cuñas que permitan desmontar aspectos discursivos parciales.

b) la puesta en evidencia de supuestos ideológicos subyacentes, de intereses políticos y económicos individuales, grupales y sectoriales que pretendan la manipulación de los marcos teóricos que sirven de soporte y guía a los discursos argumentativos y de los mismos procesos histórico - psicolingüísticos y socioculturales que estos describen y explican “(H. Vázquez 2000).

Es este concepto de crítica el que deseamos retener. Tal concepto de crítica, en Vázquez, no una implica impugnación en bloque sino un balance, evaluación de los aspectos que se aceptan y de aquellos que se rechazan. Nos referimos a un concepto o 
grupos de conceptos, y de principios generales de cada perspectiva teórica o paradigma que se disputan la construcción del conocimiento en el dominio de las ciencias sociales. Agregando una fundamentación de tal rechazo o de tal aceptación. Va generando así una concepción alternativa. Este mismo criterio, se emplea para validar los resultados de cualquier investigación de campo. De este modo, reemplaza el concepto de “objetividad" por el concepto de plausibilidad. Que se transformará, finalmente, después de haber pasado por la criba de la crítica, en una concepción "más abarcativa y plausible de la realidad interpretada y explicada y de los marcos de referencia utilizados para hacerlo y sólo en este sentido "objetiva". Vázquez ( 1994 y 2005).

Es dentro de esta línea epistemológica, aunque reteniendo los conceptos de campo, y habitus de P. Bourdieu, que planteamos nuestra perspectiva de la:

\section{Odontología Social, Preventiva y Sanitaria construida críticamente como dominio interdisciplinar.}

En este texto, y después de haber investigado este campo científico por más de veinticinco años, reelaboramos parcialmente nuestra postura epistemológica planteada en 1993. Es que el campo científico delimitado por tal articulación interdisciplinar ha recibido el impacto de disciplinas que han desarrollado su propia capacidad de articularse en tanto núcleo de convergencia interdisciplinar, como sucede con la antropología social y la etnolingüística, y aún la articulación entre ellas como nuevo núcleo de interdisciplinariedad ${ }^{3}$.

En el caso de la Odontología Social, Preventiva y Sanitaria convergen sociología, antropología social (analizando pautas culturales, patrones de conducta, el universo simbólico de la cultura, delimitando grupos sociales, las interacciones socioculturales fundamentalmente entre odontólogos / pacientes- rangos de jerarquía social y valores, entre otros aportes conceptuales, como el análisis institucional-especialmente de las instituciones prestadoras de salud), la ciencia política que contribuye,

\footnotetext{
${ }^{3}$ Consultar Hèctor Vázquez y Margot Bigot: 2006 "Convergencia lingüístico cultural en el análisis de los toba “qom”asentados en el barrio de Los Pumitas. Rosario. Argentina." IX Congreso Argentino de Antropología Social. Universidad Nacional de Misiones. Posadas del 5 al 8 de Noviembre.
} 
fundamentalmente, en el tratamiento elaboración y aplicación de políticas en todos los niveles del estado: nacional, provincial y municipal con la práctica odontológica.

Queda, entonces, muy claro que el campo científico de la Odontología Social, Sanitaria y Preventiva se construye como núcleo de interdisciplinariedad en el contexto epistemológico de las ciencias sociales.

En nuestro país, como en casi todos lo países del globo, la práctica odontológica mantiene un modelo liberal, se articula a través de profesionales que se concentran en las grandes ciudades y trabajan en forma individual. De este modo, se excluye así a grandes sectores de la población que no pueden pagar estos tratamientos. Por eso, cuando nos referimos a la Odontología Social, Sanitaria y Preventiva nos introducimos en el dominio de lo público. Son efectores de salud pública los que se esfuerzan por satisfacer estas necesidades insatisfechas de la población no contenida en el modelo de salud liberal, procurando establecer un perfil epidemiológico con vistas maximizar los logros del proceso de salud/ enfermedad/atención.

La Organización Mundial de la Salud define, en su página WEB, a la epidemiología (que en un sentido amplio puede considerase como núcleo de interdisciplinariedad entre las ciencias sociales y las biológicas) del siguiente modo: "La epidemiología es el estudio de la distribución y los determinantes de estados o eventos (en particular de enfermedades) relacionados con la salud y la aplicación de esos estudios al control de enfermedades y otros problemas de salud. Hay diversos métodos para llevar a cabo investigaciones epidemiológicas: la vigilancia y los estudios descriptivos se pueden utilizar para analizar la distribución, y los estudios analíticos permiten analizar los factores determinantes".

En el caso del procesos enfermedad/salud/ atención odontológica de la población originaria de América Latina, y de nuestro país, tema en el que nos especializamos, ha de considerarse con particular atención a la etnoepidemiologia. La etnoepidemiología realiza aportes muy considerables a nuestro campo de conocimiento. Esta disciplina, no se define solamente por utilizar métodos circunscriptos a la epidemiología clásica propia de investigaciones de estudios interculturales en salud. Sino que aporta su adopción de alternativas metodológicas, que incluyen los procesos y prácticas sociales 
relacionadas a la salud, combinando de manera competente, abordajes cualitativos y cuantitativos en una única estrategia. Al referirse a los estudios interculturales en salud Rita de Cássia P. Fernández (2003) hace notar que la epidemiología clásica ha dejado de lado en la construcción del saber epidemiológico la percepción del sujeto. En este caso, la de los actores sociales indígenas con relación al proceso salud / enfermedad / atención.

Este aspecto la cuestión indígena adquirió, paulatinamente, especial importancia en los últimos años. La suficiente como para ser incluida en estas reflexiones epistémicas y metodológicas.

La construcción de políticas públicas y la elaboración y ejecución de intervenciones sociales a través de proyectos para los pueblos indígenas son aspectos cruciales.

Sostenemos la necesidad de desarrollar políticas públicas que incluyan los derechos sociales y civiles diferenciados de los pueblos indígenas en materia de salud. Al respecto, es necesario vincular al debate sobre el proceso de salud / enfermedad / atención los aspectos políticos y éticos con relación a las estrategias de participación comunitaria. De tal modo, que los estudios sobre la salud indígena no sólo enfaticen la heterogeneidad, emergencia y dinámica, de la cultura, sino también las interacciones sociales que caracterizan el contexto interétnico, intercultural e intermedio.

En tanto "objeto" teórico construido en el análisis epistemológico de la Odontología Social, Sanitaria y Preventiva es necesario distinguir entre:

1.- Su historia interna como dominio disciplinario. Esto es, las distintas concepciones de salud bucal, las prácticas asistenciales inherentes a ellas, la formación de recursos humanos y las técnicas instrumentales de curación.

2.-Incidencias de su historia externa tales como las interacciones entre diferentes tipos de estado, el sistema político y la sociedad civil que se plasman en políticas sociales y sanitarias implementadas en cada sociedad. Y, en un plano más restringido, las incidencias de las acciones (y conflictos derivados de ellas) entre los actores sociales involucrados dentro del campo de salud/enfermedad. 


\title{
ODONTOLOGÍA SOCIAL, SANITARIA Y PREVENTIVA COMO NÚCLEO INTERDISCIPLINAR
}

\author{
5to. Núcleo de convergencia \\ POLÍTICA SANITARIA \\ $\uparrow$

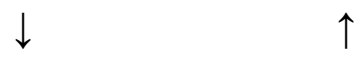 \\ 1 er Núcleo de convergencia: \\ 4 to. Núcleo de \\ Estado Liberal. Estado Benefactor. \\ Convergencia: \\ Estado Autoritario. \\ Concepto de Salud. \\ Régimen Político/Políticas Sociales. \\ 6to. Núcleo de convergencia: \\ Proceso de salud/ enfermedad/atención \\ $\uparrow$ \\ 2do. Núcleo de convergencia: \\ Historia-Sociología-Ciencia \\ Política- Antropología Social \\ 3er Núcleo de Convergencia \\ Ampliación del concepto Salud. \\ Operacionalización de conceptos

\footnotetext{
7 to. Núcleo de convergencia

MODELO DE SALUD
}

Según las modalidades de convergencia posibles

Biológico (hegemónico)

Modelo ampliado de Salud (saberes y prácticas empíricas basados en la experiencia y no en la experimentación).

\section{8 tvo. Núcleo de convergencia}




\section{MODELO DE SALUD ODONTOLÓGICO}

Primer Núcleo de convergencia: Compuesto por las conexiones entre el Sistema Político, concebido modo el modo de articulación de un Estado concreto en un momento dado de la duración histórica (Estado Liberal, Estado Benefactor, etc. Y las políticas sociales implementadas.

Segundo Núcleo de convergencia: Resulta de la teorización del Núcleo de convergencia anterior mediante el entrecruzamiento de las siguientes disciplinas: historia, sociología, antropología socio-cultural, ciencia política y ciencia jurídica en función de la teoría del Estado

Tercer Núcleo de convergencia: Ampliación del concepto de salud. Compuesto por los distintos planos de convergencia entre las siguientes disciplinas: ciencias biomédicas (las que en sí mismas se presentan como otro núcleo de interdisciplinariedad). Se trata de los diferentes enfoques de salud que se plantaron en las ciencias biomédicas tales como los causales y los sistémicos, que se entrecruzan, a su vez con lo social. Y las ciencias sociales tales como la antropología socio-cultural, la sociología, que amplían el concepto salud / enfermedad / atención aportando las cargas de significaciones, y de sentido de los actores sociales y las representaciones sociales de dichos procesos. De un modo operativo, entendemos el concepto de representación social como las imágenes del mundo (lo exterior al sujeto cognoscente) y de la vida (normas de conducta, sistemas simbólicos, creencias, valores y actitudes concebidos dinámicamente. Es decir, construidos, des-construidos y reconstruidos, según situación de momento y lugar, por los grupos sociales que comparten un acervo cultural común en constante devenir. De este núcleo de convergencia se deriva el 4 to. Núcleo de convergencia.

De la convergencia del Segundo Núcleo y el Cuarto Núcleo se deriva el 5to. Núcleo de Convergencia: la Política Sanitaria, que impulsa y legitima un Modelo de Salud Dominante: 7to. Núcleo de Convergencia, cuyas características específicas dependerán de las modalidades de intervinculación establecidas en cada Núcleo de convergencia mencionado.

Conviene destacar aquí las diferencias entre concepción de salud y modelo de salud.

Todo Modelo de Salud determina:

1.-Un tipo de práctica profesional.

2.- Un modo de atención de la salud (la organización de dicha práctica profesional). 
3.- Una concepción de la utilización de la ciencia y la tecnología.

4.- Un modo particular de delimitación de jerarquías necesidades y demandas sociales existentes.

Mientras que en el Modelo Odontológica (Octavo Núcleo de Convergencia) establecido en el aquí y en el ahora, refleja las concepciones propias del Modelo de Salud Dominante, y se refiere, fundamentalmente, a:

1.-Formación de Recursos Humanos, modelo educativo (perfil del profesional).

2.-Creación de conocimientos. Conceptuales, metodológicos y técnicos.

3.-Producción de Servicios: creación de espacios compartidos, articulación entre servicio / asistencia /comunidad.

El 6to. Núcleo de convergencia: Proceso de salud/ enfermedad/atención es generado mediante la convergencia de los núcleos $1,2,3,4$ y 5. Y es sobre condicionado por el

Modelo de Salud Odontológico (Octavo Núcleo de Convergencia) establecido en el aquí y en el ahora refleja las concepciones propias del Modelo de Salud Dominante; la Odontología Social, Preventiva y Sanitaria, concebida como Núcleo Interdisciplinar, supone un concepto ampliado de salud bucal capaz de vehiculizar las interconexiones entre problemas conceptuales, metodológicos y técnicos (en el sentido de creación de nuevos conocimientos), también la formación de Recursos Humanos en la Estrategia de Atención Primaria y de Educación para la Salud Bucal articulando espacios compartidos de interacción: docencia, asistencia, servicios y comunidad. Presentándose, por lo tanto, como modelo alternativo a las concepciones y prácticas establecidas por la economía de mercado.

\section{BIBLIOGRAFÍA}

APOSTEL, Leo; BERGER, Guy; BRIGGS, Asa y MICHAUD, Guy. 1975. PROBLEMAS DE LA ENSEÑANZA Y DE LA INVESTIGACIÓN EN LAS UNIVERSIDADES. Asociación Nacional de Universidades e Institutos de Enseñanza Superior. México.

AZCONA, María Susana 1993. "La Odontología Social como núcleo de convergencia disciplinaria", en PAPELES DE TRABAJO N³.CICEA.UNR.

AZCONA, María Susana 2002 MODELO DE ATENCIÓN ODONTOLÓGICA EN LA SALUD PÚBLICA MUNICIPAL. UNR EDITORA. 
BORDIEU, Pierre y Loica WACQANT 2005. UNA INVITACIÓN A LA SOCIOLOGÍA REFLEXIVA. Buenos .Aires: Siglo XXI Editores.

BOURDIEU, Pierre 1999 INTELECTUALES, POLÍTICA Y PODER Buenos Aires. EUDEBA.

BOURDIEU, Pierre. 1993 COSAS DICHAS. Buenos Aires Gedisa Editorial.

BOURDIEU, Pierre, CHAMBOREDON, Jean Claude y PASSERON Jean Claude. 1975 EL OFICIO DEL SOCIÒLOGO. Siglo XXI Editores.

FERNÁNDEZ GUIZZETTI, Germán. 1981 "Prolegómenos para una etnosemántica estructural" Suplemento Antropológico -Revista del Centro de Estudios Antropológicos -Asunción, Paraguay: Universidad Católica Págs. 7-113.

FERNÁNDES P.C.R. 2003 “Uma leitura sobre a perspectiva etnoepidemiológica”. En Ciência. saúde coletiva Vol.8 No.3 São Paulo.

KUHN, Thomas 1980 LA ESTRUCTURAS DE LAS REVOLUCIONES CIENTÍFICAS. Breviario del Fondo de Cultura Económica. México.

VÁZQUEZ GARCÍA, Francisco 2006 "El problema de la reflexividad en Pierre Bordieu de la Epistemología a la "Ética". En Opinión Jurídica. Vol. 5 No 10. Universidad de Medellín. Colombia.

VÁZQUEZ, Héctor 2014 "Epistemología de las ciencias sociales y las falsas dicotomías entre subjetivismo /objetivismo, explicación / comprensión, monológico / dialógica". Revista de Epistemología y Ciencias Humanas N6. ISSN: 1852-625X. Grupo Ianus. Docentes de la Universidad Nacional de Rosario y Universidad Nacional del Litoral. Pagina Web.

VÁZQUEZ, Héctor y BIGOT, Margot 2013 APORTES PARA UNA ANTROPOLOGÍA CRÍTICA Y LATINOAMERICANA. Repositorio Virtual de la Universidad Nacional de Rosario: URI http://hdl.handle.net/2133/2660.

VÁZQUEZ, Héctor 2005 ANTROPOLOGÍA EMANCIPADORA, DERECHOS HUMANOS Y PLURICULTURAIDAD. HomoSapiens Ediciones. 
VÁZQUEZ, Héctor 2000 PROCESOS IDENTITARIOS Y EXCLUSIÓN SOCIOCULTURAL-La cuestión indígena en La Argentina- Editorial Biblos. Buenos Aires.

VÁZQUEZ, Héctor 1994 LA INVESTIGACIÓN SOCIOCULTURAL -CRÍTICA DE LA RAZÓN TEÓRICA Y DE LA RAZÓN INSTRUMENTAL- Biblos, Buenos Aires.

VÁZQUEZ, Héctor 1988 ETNOLOGÍA DEL CONOCIMIENTO. Editorial Almagesto. Buenos Aires.

VÁZQUEZ, Héctor 1986 DEL INCESTO EN PSICOANÁLISISY EN ANTROPOLOGÍA. Breviario del Fondo de Cultura Económica.México.

VÁZQUEZ, Héctor 1984 SOBRE LA EPISTEMOLOGÍA Y LA METODOLOGÍA DE LA CIENCIA SOCIAL. Universidad Autónoma de Puebla, México.

WACQUANT, Loic. 2007 LOS CONDENADOS DE LA CIUDAD. GUETO, PERIFERIAS Y ESTADO. Buenos Aires, Siglo XXI Editores. Argentina 